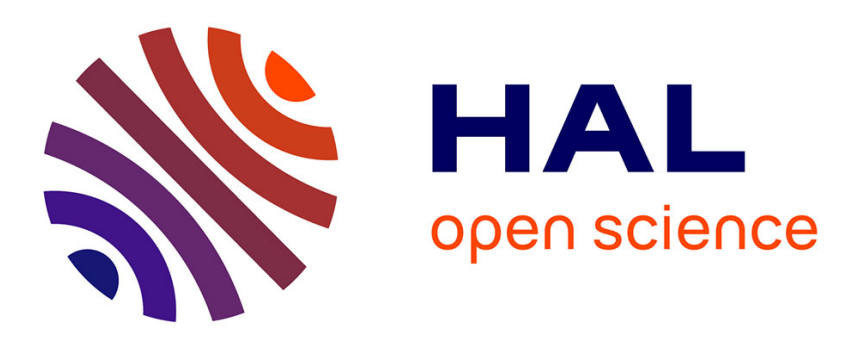

\title{
Experimental analysis on the coupled effect between thermo-optical properties and microstructure of semi-crystalline thermoplastics
}

Sinan Boztepe, Abdoulahad Thiam, Olivier de Almeida, Yannick Le Maoult, Fabrice Schmidt

\section{To cite this version:}

Sinan Boztepe, Abdoulahad Thiam, Olivier de Almeida, Yannick Le Maoult, Fabrice Schmidt. Experimental analysis on the coupled effect between thermo-optical properties and microstructure of semi-crystalline thermoplastics. ESAFORM 2016 - 19th International ESAFORM Conference on Material Forming, Apr 2016, Nantes, France. art. 020006 - 7 p., 10.1063/1.4963410 . hal-01609025

\section{HAL Id: hal-01609025 \\ https://hal.science/hal-01609025}

Submitted on 25 Mar 2019

HAL is a multi-disciplinary open access archive for the deposit and dissemination of scientific research documents, whether they are published or not. The documents may come from teaching and research institutions in France or abroad, or from public or private research centers.
L'archive ouverte pluridisciplinaire HAL, est destinée au dépôt et à la diffusion de documents scientifiques de niveau recherche, publiés ou non, émanant des établissements d'enseignement et de recherche français ou étrangers, des laboratoires publics ou privés. 


\title{
Experimental analysis on the coupled effect between thermo- optical properties and microstructure of semi-crystalline thermoplastics
}

\author{
Sinan Boztepe ${ }^{1}$, Abdoulahad Thiam², Olivier de Almeida ${ }^{1, \mathrm{a}}$, Yannick Le Maoult ${ }^{1, \mathrm{~b}}$ \\ and Fabrice Schmidt ${ }^{1, \mathrm{c}}$ \\ ${ }^{1}$ Université de Toulouse, Mines Albi, INSA, UPS, ISAE, ICA (Institut Clément Ader) Campus Jarlard, F 81013 Albi \\ Cedex 09, France \\ ${ }^{2}$ Laboratoire Interdisciplinaire Carnot de Bourgogne (ICB), UMR 6303 CNRS-Universite de Bourgogne, 21078 \\ Dijon, France \\ a) olivier.dealmeida@mines-albi.fr, ${ }^{b)}$ lemaoult@mines-albi.fr, ${ }^{c}$ fabrice.schmidt@mines-albi.fr
}

\begin{abstract}
Radiation heat transfer is the most common method used in thermoforming processes of thermoplastic polymers due to their poor thermal conductivity. Considering the fact that the thermo-optical characteristics of polymers play a major role in the efficiency of radiative heat transfer in bulk polymers, microstructure of semi-crystalline thermoplastics is one of the key factors to understand this heat transfer phenomenon in depth. In this study, a relation between the microcrystalline structure of polyolefin (PO) and its effect on the thermo-optical properties was experimentally analyzed. Information on the microcrystalline structure of the samples was obtained by determining the degree of crystallinity $\left(\mathrm{X}_{\mathrm{c}}\right)$ thanks to Differential Scanning Calorimetry (DSC). Using Fourier Transform Infrared (FTIR) spectroscopy and integrating sphere, optical characteristics of the PO samples were analyzed considering two spectrums that are in near-infrared (NIR) and middle-infrared (MIR) spectral regions respectively. The analyses showed that the degree of crystallinity has a great effect on the thermo-optical characteristics of the PO - particularly considering transmission - in NIR range. Such a coupled effect can be functionalized and adopted to develop an advanced radiative heat transfer model that may be used for addressing various problems on infrared (IR) heating of heterogeneous materials, particularly semi-crystalline thermoplastics. In the last part of the paper, a theoretical approach for consideration of the heterogeneity of semi-crystalline thermoplastics in a radiative heat transfer model was highlighted.
\end{abstract}

\section{INTRODUCTION}

The major advantage of IR radiation heating is that a portion of the radiative energy penetrates directly into the bulk of thermoplastic polymer where high heat flux densities can be used to decrease the heating time without causing thermal damage to the polymer surface [1]. Polymer structures used in thermoforming processes are heated prior to forming stage. The temperature profile at the end of the heating stage has therefore a great effect on viscoelastic properties and thus on the mechanical behavior of polymers during forming. As a consequence, it is crucially important to have an extensive knowledge of the temperature distribution throughout the bulk volume of polymer. A numerical radiative heat transfer model may be helpful either to predict the temperature distribution accurately or to optimize the heating conditions such as reducing the heating time and the input of energy. However, physical background of the radiation heat transfer into the bulk polymer needs to be understood in detail in order to develop an advanced thermal model that can address various problems in the radiation heating of thermoplastics.

In the last decade, several authors developed such a radiative thermal model and applied it successfully to various thermoplastic polymers for which the temperature distribution of the preforms heated by infrared heating was predicted closely [2]-[6]. Most of the radiative heat transfer models used in those studies adopted the BeerLambert law, assuming a homogenous and non-scattering medium for bulk material so that the radiation attenuation 
through a medium was only the function of absorption. Neglecting the scattering behavior may be valid for amorphous or semi-crystalline thermoplastics with a low degree of crystallinity. However, it may be an ill-defined assumption for the radiation heating of bulk materials with heterogeneous medium. In recent years, several efforts have been made in relation to radiation heat transfer considering the heterogeneity of heated medium. In some studies, the heterogeneous medium of thermoplastic composites has been taken into account for laser transmission welding (LTW) and automated tape placement (ATP) processes [7]-[9]. A similar approach can also be adopted for the infrared heating of bulk semi-crystalline thermoplastic polymers where both absorption and scattering factors are considered for the calculation of radiative source term inside of the bulk volume.

In our study, the effect of the microcrystalline structure on thermo-optical properties of PO was studied. As a first step, the degrees of crystallinity of the PO samples were determined using DSC under different cooling rates. Plate-like thin PO samples were prepared and cooled down under two different cooling conditions so that two groups of samples with different degrees of crystallinity were introduced. The effect of microcrystalline structure on the thermo-optical characteristics of PO was analyzed by comparing the optical properties of the samples obtained via FT-IR and integrating sphere. The analyses revealed a strong coupling between crystallinity and the thermooptical properties of PO, especially in the range of NIR. Thanks to these analyses, a relation between the microcrystalline structure and the thermo-optical properties was highlighted. By extending the analyses, a relation can be functionalized and adopted for developing an advanced radiative heat transfer model that can take into account both absorption and scattering behaviors. This point is briefly discussed in the last section of the paper.

\section{EXPERIMENTAL}

\subsection{Degree of Crystallization Measurements}

The determination of the degree of crystallinity $-\mathrm{X}_{\mathrm{c}}(\%)$ - was performed utilizing a DSC device (Perkin Elmer DSC 850). For the DSC analyses, aluminum DSC pans were prepared with the polymer pellets. $5 \mathrm{mg}$ was the average mass chosen for the analyses. At the very beginning of each test, the samples were heated up to a temperature well above the melting temperature and held $5 \mathrm{~min}$ at this temperature in order to delete the previous thermal history of the material (e.g. processing conditions, degree of crystallinity, service temperature, etc.). Afterwards, the samples were cooled down at different cooling rates $\left(1\right.$ to $\left.100^{\circ} \mathrm{C} / \mathrm{min}\right)$ and the $\mathrm{X}_{\mathrm{c}}(\%)$ values corresponding to each adopted cooling rate were calculated on the melting curves performed systematically at $10^{\circ} \mathrm{C} / \mathrm{min}$. Apart from obtaining information on degree of crystallinity at different cooling rates, the crystallization $\left(\mathrm{T}_{\mathrm{c}}\right)$ and melting temperature $\left(\mathrm{T}_{\mathrm{m}}\right)$ of the polymer were also determined thanks to the DSC analyses.

For the determination of the degree of crystallinity, the enthalpy of crystallization was calculated by integrating the melting peak $\left(\Delta \mathrm{H}_{\mathrm{c}}\right)$ on DSC curves. The degree of crystallinity for each cooling rate was then determined thanks to Equation 1:

$$
X_{c}(\%)=\frac{\Delta H_{c}}{\Delta H_{c}^{0}}(\%)
$$

Enthalpy of the polymer that has $100 \%$ degree of crystallization $\left(\Delta \mathrm{H}_{\mathrm{c}}{ }_{\mathrm{c}}\right)$ was chosen from literature.

\subsection{Optical characterization}

In order to analyze the effect of the degree of crystallinity on the thermo-optical properties of the polymer, a method different from using pellets - as utilized in the DSC analyses - was followed. This step was employed since it was necessary to obtain void-free specimens for an accurate optical characterization. For the purpose of fabricating void-free samples, $2.2 \mathrm{~mm}$ thick plate-like thin samples were prepared using injection molding. Then, these samples that will be used in optical characterization tests were squeezed under the adopted temperature well above the $T_{m}$ to reduce their thickness. Under the same compression level and temperature, samples with the same thickness $(0.5 \mathrm{~mm})$ were prepared and grouped based on two different cooling rates for the purpose of introducing different degrees of crystallinity amongst the samples. The samples in two different groups were cooled down under quenching in water and still air conditions respectively. Therefore, two groups of samples with quite different degrees of crystallinity, but geometrically identical, were prepared. Thanks to this step, the difference in the 
measurement of transmission, reflection and absorption factors of both samples group could be related to the degree of crystallinity, but not to the sample thickness.

The optical characteristics of the samples were determined over the range of NIR $(0.8-2 \mu \mathrm{m})$ and MIR $(2-25 \mu \mathrm{m})$. Transmission factor $\left(\mathrm{T}_{\lambda}\right)$ of the PO samples with different degrees of crystallinity was determined employing FT-IR spectrometer (Bruker Vertex 70) where also their reflection factor $\left(\mathrm{R}_{\lambda}\right)$ was determined via mounting the integrating sphere as an external device on the spectrometer. The physical meanings of $T_{\lambda}$ and $R_{\lambda}$ are the ratio of the total amount of transmitted $\left(\Phi_{\lambda}{ }^{t}\right)$ and reflected $\left(\Phi_{\lambda}{ }^{r}\right)$ radiative flux over incident flux $\left(\Phi_{\lambda}{ }^{i}\right)$ respectively [10] as also stated in Equation 2. The incident flux $\left(\Phi_{\lambda}{ }^{t}\right)$ is the initial excited photon flux sent by the spectrometer.

$$
T_{\lambda}=\frac{\phi_{\lambda}^{t}}{\phi_{\lambda}^{i}} \quad \text { and } \quad R_{\lambda}=\frac{\phi_{\lambda}^{r}}{\phi_{\lambda}^{i}}
$$

\section{RESULTS AND DISCUSSION}

The DSC analyses were done performing consecutive heating and cooling scans. Except from the initial heating run that was done to delete the thermal history of the each sample, all the heating and cooling runs were recorded throughout the analyses. Figure 1 illustrates one of the heating/cooling scan of the DSC analyses that can represent all the other scans during the analyses. In the figure, the two curves with upward and downward peaks represent the heating and cooling runs respectively. As it is seen in the figure, the upward peak - which is an endothermic reaction - provides information on the melting point of the sample. The melting temperature $\left(\mathrm{T}_{\mathrm{m}}\right)$ of the sample was determined around $130^{\circ} \mathrm{C}$. In addition, the melting enthalpy of each scan was calculated based on the integrated area of this peak, and the degree of crystallinity of each scan that is function of the corresponding cooling rate was determined. As a different cooling rate was applied during each scan, the heating run of each subsequent scan showed a slight difference compared to the previous one, as a consequence of the different degree of crystallinity. Similarly, the downward peak - an exothermic reaction - provides information on the crystallization temperature $\left(\mathrm{T}_{\mathrm{c}}\right)$ during each cooling run and $\left(\mathrm{T}_{\mathrm{c}}\right)$ was estimated around $115^{\circ} \mathrm{C}$.

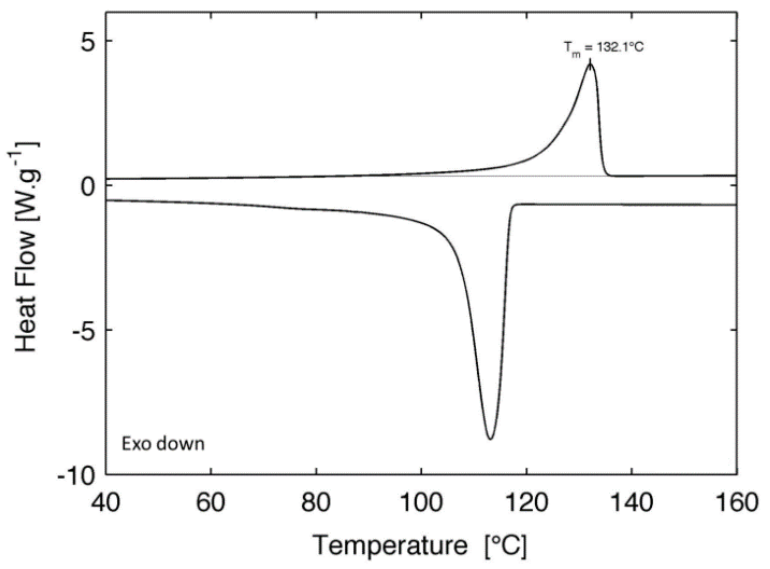

FIGURE 1. Typical heating / cooling scan obtained during the DSC analyses of polyolefin samples at cooling rate of $20^{\circ} \mathrm{C} / \mathrm{min}$

The DSC analyses confirmed that the polyolefin-based thermoplastic used in this study shows a degree of crystallinity higher than $60 \%$ considering the cooling rate between 1 and $100^{\circ} \mathrm{C} / \mathrm{min}$ and these results are in good agreement with literature [11]. Figure 2 shows the relation between the adopted cooling rate and the determined degree of crystallinity of the pellets in the DSC analyses. 


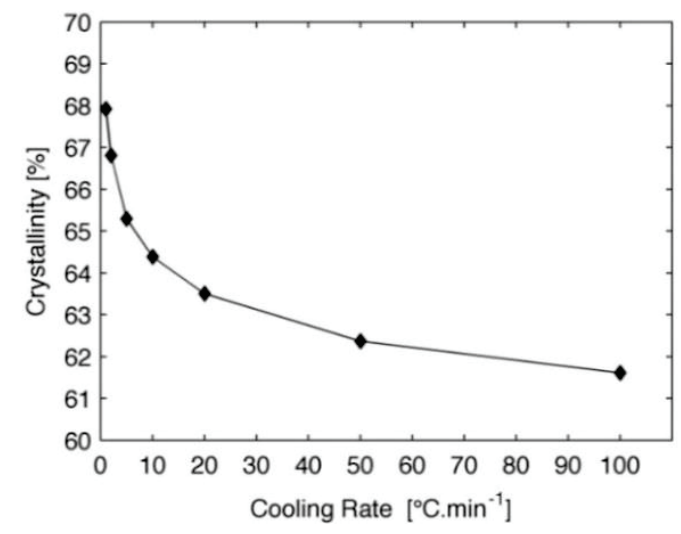

FIGURE 2. Relation between the adopted cooling rate and the determined degree of crystallinity of the PO samples.

As aforementioned, two groups of plate-like samples were prepared using two different cooling methods for the analyses of the optical characteristics. Utilizing DSC, the degree of crystallinity was obtained for the plate-like thin samples. The samples quenched in water and cooled down in still air showed a crystallinity of 58\% and $63 \%$ respectively.

Transmission behavior of the samples from both groups was measured using FT-IR spectrometer. Figure 3 (a) and (b) illustrate the transmission factor of different samples over the spectral ranges of $0.8-2 \mu \mathrm{m}$ and $2-25 \mu \mathrm{m}$, which are in the NIR and MIR regions respectively [12], [13]. The peak wavelength of emission $\left(\lambda_{\max }\right)$ of a typical IR heater is in the spectral range around $1-1.2 \mu \mathrm{m}$, that varies regarding to IR set temperature at which $96 \%$ of the emission occurs between $0.5 \lambda_{\max }$ and $5 \lambda_{\max }$. As it is seen in Figure 3 (a), even an increase of $5 \%$ of the degree of crystallinity causes a decrease of nearly $50 \%$ of the transmission factor in the infrared spectral range around $1 \mu \mathrm{m}$ that is close to the maximum emission wavelength of a typical IR heater. Conversely, the same trend was not seen in the MIR range, where only a slight difference of 3-5\% was observed between the samples in the spectral range of 7 $15 \mu \mathrm{m}$ (Figure 3(b)). It is clearly evident that a slight variation in the degree of crystallinity of the samples has great effect on its thermo-optical characteristics in a specific spectrum in the NIR range. This may be explained by the relation between wavelength of transmitted light and scatterer in the medium represented by spherulite or lamellae size in the microcrystalline structure of the PO samples. Therefore it can not be neglected in case of building a thermal model for IR heating assisted thermoforming processes of the used polyolefin-based material.

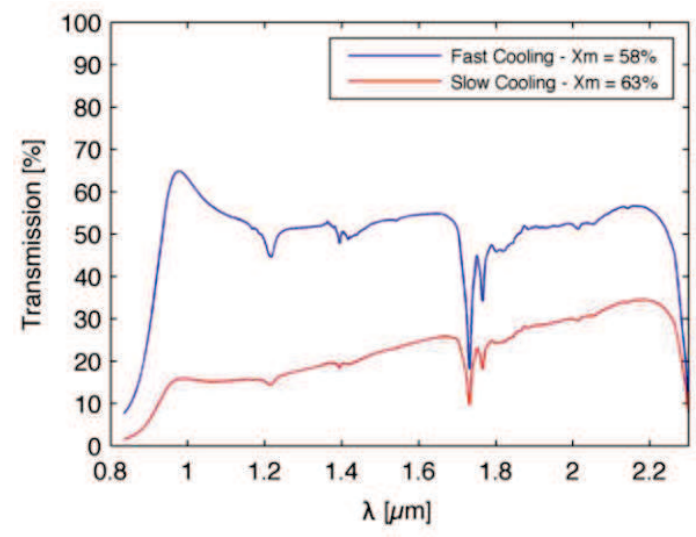

(a)

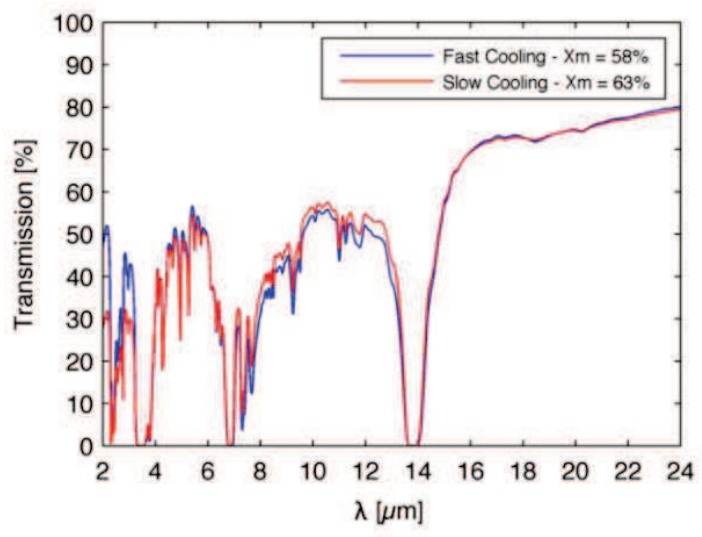

(b)

FIGURE 3. Transmission factor of the samples with different degrees of crystallinity in the range of NIR (a) and MIR (b).

The measurements on the reflection factor using integrating sphere showed that an inverse trend regarding the transmission behavior. As illustrated in Figure 4, the higher the degree of crystallinity, the higher the reflection factor in NIR range (a) whereas no effect was observed in the MIR range (b). The reflection factors of both the samples show a decreasing trend with an increase in wavelength over the NIR range. The difference between the reflection factors of the two samples is around 3\% in the NIR range. 


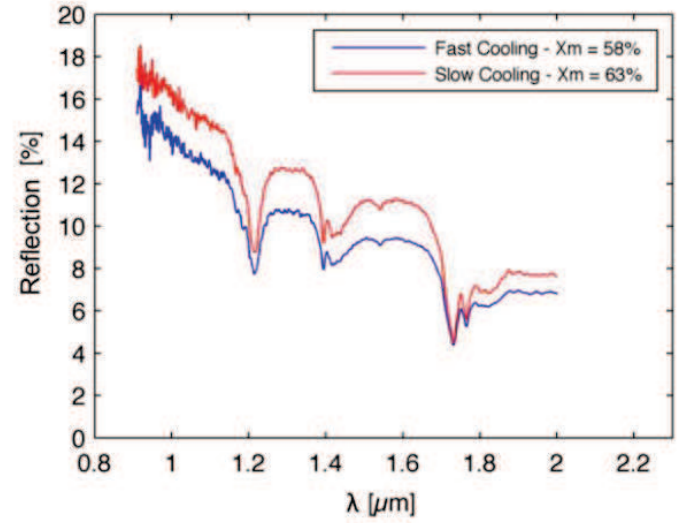

(a)

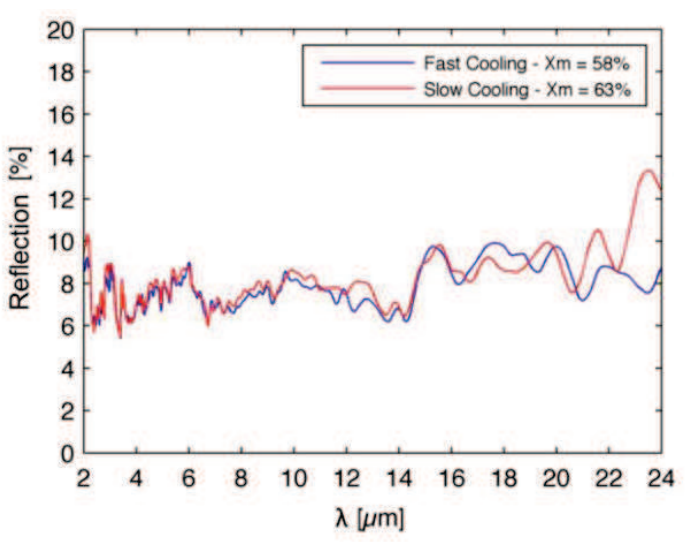

(b)

FIGURE 4. Reflection factor of the samples with different degrees of crystallinity in the range of NIR (a) and MIR (b).

The measurement of the reflection factor may only be related to the reflected flux of the sample surface but not to the opacity of the samples. As illustrated in Figure 3, the transmission factor of the sample with high degree of crystallinity (cooled down slowly) has a much lower transmission factor. The absorption characteristics combined with the transmission and reflection may provide an extended insight into opacity of the samples. Under the law of energy conservation, it can be formulated that the incident radiative flux $\left(\Phi_{\lambda}{ }^{1}\right)$ equals to the sum of transmitted $\left(\Phi_{\lambda}{ }^{\mathrm{t}}\right)$, reflected $\left(\Phi_{\lambda}{ }^{\mathrm{r}}\right)$ and absorbed $\left(\Phi_{\lambda}{ }^{\mathrm{a}}\right)$ radiative flux. It should be noted that the attenuated radiation intensity in the medium was calculated based on Beer-Lambert law under an assumption of homogenous non-scattering cold medium. Under these assumptions, the relation between the attenuation of the radiative flux at a depth of ' $\mathrm{z}$ ' through the thickness of the samples can be formulated as spectral intensity of radiative flux $\left(\mathrm{I}_{\Delta \lambda}(\mathrm{z})\right)$ stated in Equation 3:

$$
I_{\Delta \lambda}(z)=I_{\Delta \lambda}^{o} \cdot e^{\left(-\kappa_{\Delta \lambda} \cdot z\right)} \text { with; } \quad \kappa_{\Delta \lambda}(T)=\frac{\int_{\lambda_{1}}^{\lambda_{2}} \kappa_{\lambda} I_{\lambda}^{o}(T) d \lambda}{\int_{\lambda_{1}}^{\lambda_{2}} I_{\lambda}^{o}(T) d \lambda}
$$

$\kappa_{\Delta \lambda}(T)$ is the absorption coefficient integrated over the spectral range $(\Delta \lambda)$ for a given temperature $(T)$. In the current study, the incident emitted flux was calculated for an emitter at a temperature of $2400 \mathrm{~K}$ in the range $[0.64$ $6 \mu \mathrm{m}]$ at which $\lambda_{\max }$ was determined as $1.2 \mu \mathrm{m}$. At the adopted temperature, two different integrated absorption coefficients were calculated for the two different transmission behaviors obtained through the optical tests. The cumulative absorbed flux through the thickness of two groups of samples was illustrated in Figure 5. As seen in the figure, due to the two different absorptions, the higher degree of crystallinity causes to attenuate all the incident flux in a depth around $0.25 \mathrm{~mm}$ whereas the full attenuation is around $3 \mathrm{~mm}$ for the samples with lower crystallinity. This can be explained by the prolonged optical path of the radiation passing through the medium due to scattering [14] and cause to increase absorption in an equivalent depth, as also reported in [15].

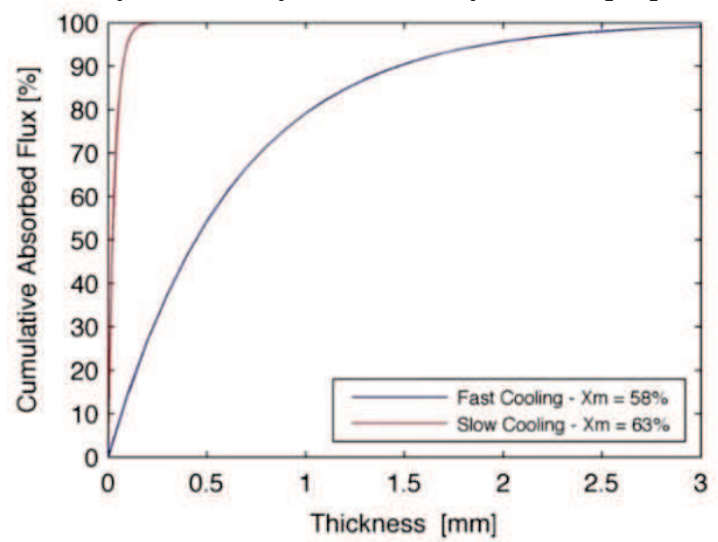

FIGURE 5. Cumulative absorbed flux through the thickness of the samples with a different degree of crystallinity. 
Therefore, Equation 3 with the assumption of a homogenous medium may not be sufficient to model the radiative heating of semi-crystalline thermoplastics with a high degree of crystallinity, as the scattering behavior is neglected. In order to model accurately the radiative heating stage of such a polymer, both the scattering and absorption effects have to be taken into account. Radiative Transfer Equation (RTE), as being a theoretical backbone of radiative heat transfer problems, may solve such a coupled effect. By adopting the integrated form of radiative transfer equation (RTE) [16]:

$$
I\left(\tau_{\lambda}, \Omega\right)=I_{\lambda}(0, \Omega) \cdot e^{\left(-\tau_{\lambda}\right)}+\int_{\tau_{\lambda}^{*}=0}^{\tau_{\lambda}(S)} \hat{I}_{\lambda}\left(\tau_{\lambda}^{*}\right) e^{-\left(\tau_{\lambda}-\tau_{\lambda}^{*}\right)} d \tau_{\lambda}^{*} \quad \text { with; } \quad \tau_{\lambda}(S)=\int_{S^{*}=0}^{S}\left(\kappa_{\lambda}(S)+D_{\lambda}(S)\right) d S
$$

Advanced factors in radiative transfer along the direction of $\mathrm{S}$ and solid angle $(\Omega)$ that are integrated over the optical thickness from $\tau_{\lambda}^{*}=0$ to $\tau_{\lambda}=\tau_{\lambda}(S)$ can be taken into account for a numerical model. The term $\tau_{\lambda}^{*}$ is a dummy optical variable of integration along the $\mathrm{S}$ direction. In Equation 4, the first term is similar to the BeerLambert law as expressed in Equation 3 that represents an attenuated initial intensity that reaches at optical depth $\tau_{\lambda}$. The second term is the intensity at $\tau_{\lambda}$ caused by emission and incoming scattering in medium. The sum of absorption $\left(\kappa_{\lambda}\right)$ and scattering coefficient $\left(D_{\lambda}\right)$ is defined as an extinction coefficient $\left(\beta_{\lambda}\right)$ at which the optical depth is function of both scattering and absorption effects. In addition, the source function $\left(\hat{I}_{\lambda}\left(\tau_{\lambda}, \Omega\right)\right)$ represents the part of an increase in intensity by absorption and in-scattering that is also function of single scattering albedo ( $\left.\mathrm{w}_{\lambda}\right)$ and optical thickness. The single scattering albedo is assumed if the photon is scattered only once along its original incident direction. The single scattering albedo, as being a ratio of scattering to extinction coefficient, and its relation to the source function can be stated with the formula at below [16]:

$$
\hat{I}_{\lambda}\left(\tau_{\lambda}, \Omega\right)=\left(1-w_{\lambda}\right) I_{\lambda b}\left(\tau_{\lambda}\right)+\frac{w_{\lambda}}{4 \pi} \int_{\Omega_{i}=0}^{4 \pi} I_{\lambda}\left(\tau_{\lambda}, \Omega_{i}\right) \Phi_{\lambda}\left(\Omega, \Omega_{i}\right) d \Omega_{i} \quad \text { with; } \quad w_{\lambda}=\frac{D_{\lambda}}{\kappa_{\lambda}+D_{\lambda}}=\frac{D_{\lambda}}{\beta_{\lambda}}
$$

where $I_{\lambda b}$ and $\Phi_{\lambda}$ are blackbody intensity and phase function respectively. Considering the optically heterogeneous nature of semi-crystalline thermoplastics, the micro-crystalline structure of a studied material and its effect on thermo-optical properties may be functionalized and $\mathrm{W}_{\lambda}$ can have a value between 0 and 1 regarding the scattering and absorption characteristics of a heated medium.

\section{CONCLUSIONS}

In the current study, the effect of the microcrystalline structure on the thermo-optical properties of polyolefin was experimentally analyzed. The analyses demonstrated that the effect of degree of crystallinity on the transmission was significant considering the range of NIR, whereas a slight effect was observed in the MIR range. Even an increase of 5\% of the degree of crystallinity caused a decrease of nearly $50 \%$ of the transmission factor in the infrared spectral range around $1 \mu \mathrm{m}$. Considering IR heating assisted thermoforming processes, it is crucial to take into account this coupled effect as the maximum emission wavelength of a typical IR heater is close to this spectral range. In addition, the crystallinity does not only affect the transmission through the material but also causes a change in the reflection as it was also determined that a slight change in crystallinity is somewhat effective on the reflection factor in NIR range. As the change in the crystallinity degree has much greater impact in the NIR range than the MIR range, the relation between the wavelength of the transmitted light and the spherulite or lamellae size in the micro-crystalline structure may have a key role to change the scattering behavior and therefore the thermooptical parameters. It may therefore be concluded that the change in the thermo-optical characteristics of the PO samples may not be only addressed by the crystallinity itself but also its micro-crystalline morphology characterized by spherulite and lamellae structures.

These findings clearly show that the microstructure and thermo-optical properties of highly crystalline thermoplastics are needed to be coupled in order to develop an advanced IR heating numerical model. In this study, the strong coupling between the crystallinity and thermo-optical properties of the examined polyolefin, and a preliminary approach for developing a numerical model assuming a heterogeneous medium was highlighted. In our 
future work, this coupled relation will be studied further and a constitutive model will be established based on the physical background of the scattering and absorption behaviors that are strongly related to the microcrystalline morphology of semi-crystalline thermoplastics. In addition, as microcrystalline morphology of thermoplastics may change under heating process, temperature dependent morphology and its effect on the thermo-optical properties will be considered. Therefore, in the next work, heterogeneous and scattering medium conditions that may be a function of both the microcrystalline morphology and temperature will be taken into account for developing an advanced radiation heat transfer model.

\section{REFERENCES}

1. G. Burkhardt, U. Hüsgen, M. Kalwa, G. Pötsch, and C. Schwenzer, "Plastics Processing, 1. Processing of Thermoplastics," in Ullmann's Encyclopedia of Industrial Chemistry, edited by Giuseppe Bellussi et al. (Wiley-VCH Verlag GmbH \& Co. KGaA, Weinheim, 2011), pp. 155-192.

2. F. Schmidt, J. Mater. Process. Technol. 143-144, 225-231 (2003).

3. M. Bordival, F. M. Schmidt, Y. L. Maoult, and V. Velay, Polym. Eng. Sci. 49, 783-793 (2009).

4. S. Monteix, F. Schmidt, Y. Le Maoult, R. B. Yedder, R. W. Diraddo, and D. Laroche, J. Mater. Process. Technol. 119, 90-97 (2001).

5. J. M. P. Coelho, M. A. Abreu, and F. Carvalho Rodrigues, Polym. Test. 23, 307-312 (2004).

6. F. Becker and H. Potente, Polym. Eng. Sci. 42, 365-374 (2002).

7. A. C. A. Asséko, B. Cosson, F. Schmidt, Y. L. Maoult, and E. Lafranche, Infrared Phys. Technol. 72, 293299 (2015).

8. M. Chen, G. Zak, and P. J. Bates, J. Mater. Process. Technol. 211, $43-47$ (2011).

9. C. M. Stokes-Griffin and P. Compston, Opt. Lasers Eng. 72, 1-11 (2015).

10. E. F. Zalewski, "Radiometry and photometry," in Handbook of Opics. 2nd ed., edited by M.Bass et al, (McGraw-Hill, New York, 1995) vol.2, pp 24.1 -24.50.

11. A. S. M. International and S. Lampman, Characterization and Failure Analysis of Plastics. (ASM International, Materials Park, 2003), pp. 36.

12. R. A. Viscarra Rossel, D. J. J. Walvoort, A. B. McBratney, L. J. Janik, and J. O. Skjemstad, Geoderm. 131, 59-75 (2006).

13. E. N. Lewis and L. H. Kidder," Technologies and Practical Considerations for Implementing Near-Infrared Chemical Imaging," in Raman, Infrared, and Near-Infrared Chemical Imaging, edited by S. Sasic and Y. Ozaki (John Wiley \& Sons Inc., Hoboken, 2011), pp. 75-91.

14. N. Wolf, D. Petring, A. L. Boglea, A. Roesner, U. A. Russek, F. Sari, L. Bosse, F. Schmitt, K. Klages, A. Olowinsky, "Joining," in Tailored Light 2: Laser Application Technology, edited by R. Poprawe (Springer Science \& Business Media, Berlin, 2011), pp. 265-341.

15. M. Geiger, T. Frick, and M. Schmidt, Prod. Eng. 3, 49-55 (2009).

16. J. R. Howell, M. P. Menguc, and R. Siegel, Thermal Radiation Heat Transfer, 6th ed. (CRC Press, Baton Rouge, 2015). 